\title{
Hipk is required for JAK/STAT activity and promotes hemocyte-derived tumorigenesis
}

Running Title: Hipk promotes JAK/STAT signaling

\author{
Jessica A. Blaquiere, Nathan B. Wray and Esther M. Verheyen \\ Department of Molecular Biology and Biochemistry \\ Centre for Cell Biology, Development and Disease \\ Simon Fraser University, Burnaby, B.C Canada \\ Corresponding author: everheye@sfu.ca
}

Keywords: Hipk, JAK/STAT, Stat92E, Drosophila, hemocyte, tumor 
Summary Statement: Loss of hipk impairs JAK/STAT activity in multiple tissue types and elevated Hipk leads to the formation of blood cell tumors in Drosophila.

\section{Abstract:}

Dysregulation of key signaling molecules and pathways are causative of many Human diseases and cancers. A point mutation in the Drosophila Janus kinase (called hop) causes constitutive activation of the JAK/STAT pathway and results in blood cell tumours. We provide robust genetic evidence that Hipk is required for endogenous JAK/STAT activity. Overexpression of Hipk can phenocopy the effects of overactive JAK/STAT mutations and lead to melanized tumors and loss of Hipk can suppress the effects of hyperactive JAK/STAT. Furthermore, Hipk expression in blood cell progenitors causes tumors. PLA experiments show that Hipk can interact with the pathway effector Stat92E. Together our results show that Hipk is a novel factor required for effective JAK/STAT signaling.

\section{Introduction:}

Drosophila is a useful model to study evolutionarily conserved signaling pathways that are used reiteratively during development, as well as for modeling diseases, such as leukemia. Dysregulation of the JAK/STAT pathway has been linked to leukemia, myeloproliferative neoplasms, and solid tumors in flies and vertebrates (Amoyel et al., 2014; Dearolf, 1998; Jones et al., 2005; Lacronique, 1997; Levine et al., 2005). In Drosophila, the core components of the pathway include the Unpaired ligands (Upd, Upd2, Upd3), the Domeless receptor (Dome), the JAK homolog Hopscotch (Hop), and the transcription factor Stat92E (reviewed in Chen et al., 2014). Upon cascade stimulation, Stat92E becomes phosphorylated, dimerizes, and travels to the nucleus to regulate JAK/STAT target genes. JAK/STAT mutations are heavily correlated with tumor invasiveness and lethality (Hanratty and Dearolf, 1993). hop ${ }^{\text {Tum-I }}$ is a dominant mutation resulting in a hyperactive Hop kinase that leads to constitutive activation of the pathway (Harrison et al., 1995). Similar activating JAK2 mutations are commonly seen in vertebrate cancers (Jones et al., 2005; Kralovics et al., 2005).

Homeodomain-interacting protein kinase (Hipk in Drosophila, Hipk1-4 in 
vertebrates) regulates numerous conserved signaling pathways (Chen and Verheyen, 2012; Lee et al., 2009a; Lee et al., 2009b; Poon et al., 2012; Rinaldo et al., 2008; Swarup and Verheyen, 2011). Interestingly, Hipk overexpression results in tumor-like formations similar to those seen in hop ${ }^{\text {Tum-l }}$ flies, prompting our investigation into Hipk's role in the JAK/STAT pathway. Indeed, reducing hipk suppressed the severity of the hop ${ }^{\text {Tum-I }}$ phenotype. Further, we provide evidence that Hipk is required for JAK/STAT activity in a kinase-dependant manner and that Hipk and Stat92E interact in vivo. Our data indicate a novel role for Hipk in regulating JAK/STAT activity in endogenous and tumorous conditions.

\section{Results and Discussion:}

\section{Hipk induces hemocyte-derived melanotic tumors}

We observed that Hipk induces pigmented masses, a phenotype resembling flies with overactive JAK/STAT signaling (Hanratty and Ryerse, 1981; Luo et al., 1995). Overexpression of hipk with $d p p-G A L 4$ (dpp>HA-hipk $\left.{ }^{3 M}+2 x G F P\right)$ caused the formation of pigmented tumors in the larval stages of development (Fig. 1B). These masses were not due to cell death, since they persisted when cell death was blocked with P35 (Fig. 1C) (Hay et al., 1994). Melanotic tumors induced by hop ${ }^{\text {Tum-I }}$ arise due to overamplification and melanization of hemocytes, fly hematopoietic cells (Hanratty and Ryerse, 1981). Therefore, we next tested whether hipk could cause tumors when overexpressed in the circulating hemocytes and lymph gland using hemolectin-GAL4 (hml-GAL4) (Sinenko and Mathey-Prevot, 2004). 91.7\% of hml>HA-hipk ${ }^{3 M}$ flies exhibited at least one melanotic tumor, with the average being 3-4 tumors (Fig. 1E,F), compared to $0 \%$ of $h m />$ GFP flies (Fig. 1D).

We hypothesized that, similar to hop ${ }^{\text {Tum-I }}$, Hipk may increase the number of circulating hemocytes. We tested this by isolating the total hemolymph from third instar (L3) larvae (Fig. S1C), and determined that the mean number of hemocytes in each $h m l>H A-h i p k^{3 M}+$ GFP sample was 197, compared to 39 per $h m l>2 \times G F P$ sample (Fig 1G-I). $h m l>H A-h_{i p k^{3 M}+G F P}$ samples often contained large aggregated clusters of hemocytes (Fig. S1B), a phenotype found in hop ${ }^{\text {Tum-l }}$ hemocyte samples (Luo et al., 
1997). These data suggest that the tumors induced by Hipk, like the ones induced by hop ${ }^{\text {Tum-l }}$, are derived from the hemocytes.

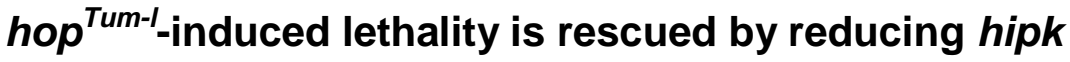

Extensive characterization of the hop ${ }^{\text {Tum-l }}$ allele by others has shown that it can be utilized in lethality and tumor frequency assays to help in identifying novel JAK/STAT pathway components and regulators (Chen et al., 2014). We tested whether hipk could modify the hop ${ }^{\text {Tum-l }}$ lethality phenotype (see Fig. 2F-H for ranking examples) (Rawlings et al., 2004; Yan and Luo, 1996). hop ${ }^{\text {Tum-l; ; } M K R S / T M 6 B}$ animals raised at $29^{\circ} \mathrm{C}$ were larval or early pupal lethal (Fig. 2A,B); $91 \%$ of pupae died in the early pupal stage, $9 \%$ died in the late pupal stage, and $0 \%$ of adults eclosed (Fig. $2 \mathrm{E}$ ). Heterozygous reduction of hipk (hop ${ }^{\text {Tum-l }}$;; hipk ${ }^{4} / T M 6 B$ ) suppressed this phenotype (Fig. $2 \mathrm{C}, \mathrm{D}) ; 23 \%$ of pupa died during early pupal development, and $76 \%$ died as pharate adults and $1 \%$ were able to eclose (Fig. 2E). Thus we infer that hipk is a positive regulator of the pathway since reducing hipk suppressed phenotypes caused by overactive JAK/STAT.

Since hop ${ }^{\text {Tum-l }}$ tumors derive from hemocytes, we asked whether reduction of hipk within the hemocytes could rescue hop ${ }^{\text {Tum-l }}$ lethality at $29^{\circ} \mathrm{C}$. We expressed UAS-

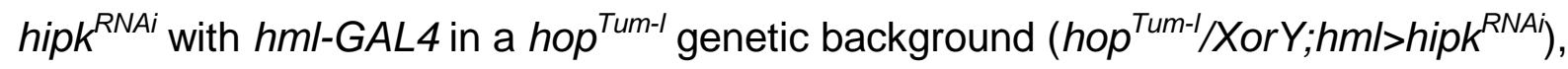
but did not observe a significant suppression (Fig. S2A-D). We reasoned that we were unable to obtain a rescue, possibly due to a combination of the strength of the hop $p^{\text {Tum-l }}$ phenotype at $29^{\circ} \mathrm{C}$, and weakness of $h m l>U A S-h i p k^{R N A i}$. hop ${ }^{\text {Tum-l }}$ is temperature sensitive, yielding a more severe phenotype at $29^{\circ} \mathrm{C}$ than at $25^{\circ} \mathrm{C}$. We tested whether loss of hipk within hemocytes could rescue the hop ${ }^{\text {Tum-l }}$ phenotype at $25^{\circ} \mathrm{C}$. hop ${ }^{\text {Tum- }}$ I/Y;hml-GAL4/+ flies raised at $25^{\circ} \mathrm{C}$ exhibited a range of tumor frequencies: $15 \%$ of flies had more than 5 small to large tumors (class 1 ; Fig. S2E,H), $50 \%$ of flies had more than 5 small to medium tumors (class 2; Fig. S2F,H), and $35 \%$ of flies had less than 5

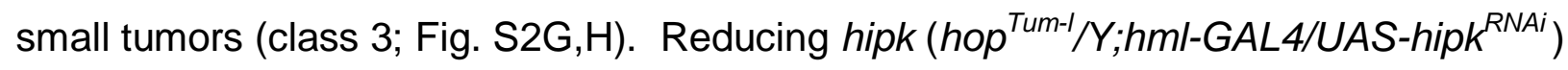
rescued the severity of $h o p^{\text {Tum-l }}$ induced tumors; we observed $0 \%$ of flies in class 1 , $29 \%$ of flies in class 2 , and $71 \%$ of flies in class 3 (Fig. S2H). We conclude that hipk is required for the full severity of the hop ${ }^{\text {Tum-l }}$ phenotype. 


\section{hipk promotes JAK/STAT signaling downstream of upd}

Cumulatively, our results suggest that hipk promotes JAK/STAT within the hemocytes. To further test hipk's role with the JAK/STAT pathway we utilized the Stat92E transcriptional reporter 10xStat92E-GFP in L3 imaginal discs, which provides an accurate representation of endogenous pathway activity (Bach et al., 2007). Loss of hipk in somatic clones led to significant cell-autonomous reductions in 10xStat92E-GFP expression in wing and eye-antennal imaginal discs (Fig. 3B, Fig. S3B), while $d p p>H A-$ hipk ${ }^{3 M}$ wing discs raised at $25^{\circ} \mathrm{C}$, and at $29^{\circ} \mathrm{C}$, caused elevated 10xStat92E-GFP (Fig. 3E, Fig. S3C). We found that expressing UAS-HA-hipk ${ }^{W T}$-attP4O within hipk ${ }^{4}$ clones could restore, and in some instances elevate 10xStat92E-GFP levels (Fig. 3C) indicating that the effect we see is due to Hipk expression. Next we tested if Hipk's kinase activity was crucial for this effect on the JAK/STAT reporter. Expression of kinase dead Hipk (UAS-HA-hipk ${ }^{K D}$-attP40) within hipk ${ }^{4}$ MARCM clones was unable to promote expression of 10xStat92E-GFP (Fig. 3D). Further support that Hipk is a positive regulator of JAK/STAT, we find that heterozygosity for hipk enhanced the small eye phenotype seen in outstretched (os; or Unpaired upd) mutants (Fig. S3F-J). Together, these results indicate that Hipk promotes JAK/STAT activity and is required for the proper output of the pathway in a cell autonomous fashion and kinasedependent manner.

To determine whether hipk promotes JAK/STAT activity upstream or downstream of Upd, we examined upd-lacZ expression in imaginal discs upon modulation of hipk. upd-lacZ is not prevalent in the L3 wing disc (Fig. 3F), but is expressed in cells at the posterior center of the L3 eye disc (Fig. $3 \mathrm{H})$. Loss of hipk in the eye disc did not alter upd expression (Fig. 3I) and upd remained unchanged in $d p p>H A-h i p k^{3 M}$ wing discs raised at $25^{\circ} \mathrm{C}$, conditions under which $10 x$ Stat92E-GFP is normally induced by Hipk (Fig. 3G, E'). However, dpp>HA-hipk ${ }^{3 M}$ wing discs raised at $29^{\circ} \mathrm{C}$ exhibited a small amount of upd up-regulation (Fig. S3D). While we observed that high levels of Hipk induce mild ectopic upd in the wing disc (Fig. S3D), past studies have shown that hipk promotes multiple signaling pathways, and this could represent 
an indirect up-regulation of upd. With this in mind, we conclude that hipk promotes JAK/STAT activity downstream of upd.

\section{Hipk physically interacts with Stat92E}

Next we attempted to elucidate how Hipk was mediating its effects on the JAK/STAT pathway. Though partly cytoplasmic, Hipk primarily localizes to the nucleus (Kim et al., 1998). Because Stat92E is also found in the nucleus, we began testing for a physical Hipk-Stat92E interaction. We utilized a proximity ligation assay (PLA), which can detect whether two proteins of interest are less than 40nm apart in vivo (Söderberg et al., 2006). In dpp>HA-hipk ${ }^{1 M}+M Y C$-Stat92E wing discs we probed with HA and MYC antibodies and observed a positive PLA reaction (Figure 4C,D). While these are ectopically expressed proteins, it is clear from the mild disc phenotype that the expression levels are not extreme and that the interaction is unlikely to be due to protein saturation. Negative control discs (dpp>HA-hipk $\left.{ }^{1 M}+G F P\right)$ that were probed against GFP and HA did not yield a PLA signal (Fig. 4A). These data suggest that Hipk and Stat92E come into close proximity in wing disc cells, though we cannot exclude the possibility that Hipk and Stat92E may come into close proximity as part of a protein complex

In summary, we present novel evidence that Hipk is an essential regulator of the JAK/STAT pathway in normal and tumorigenic processes and acts downstream of upd in a kinase-dependant manner. Output of JAK/STAT is perturbed upon loss of hipk, and increased Hipk induces hematopoietic tumors and elevated JAK/STAT activity. Further, we provide in vivo data that suggests a physical interaction between Hipk and Stat92E. Reports in vertebrate studies have found that an activated version of Hipk2 phosphorylates Stat3 (Matsuo et al., 2001; Ohtsu et al., 2007), and that Hipk2 is a potential drug target in treating Acute Myeloid Leukemia (Fleischmann et al., 2014). Future studies with help determine the precise mechanism of Hipk's role in this pathway and could ultimately lead to new therapeutics used to treat human cancers.

\section{Methods:}

Genetic crosses and fly stocks: Flies were raised on standard media. Crosses were 
raised at $25^{\circ} \mathrm{C}$ unless otherwise noted. 10xstat92E-GFP (BL\#26197) (Bach et al., 2007), UAS-eGFP (BL\#5431), UAS-eGFP (BL\#5430), UAS-P35 (BL\#5072) (Hay et al., 1994), hsflp ${ }^{122} ; ;$ Ubi-RFP,FRT79 (made from BL\#34498), $y^{1} v^{1} h o p^{T u m} / F M 7 c$ (BL\#8492; referred to as hop ${ }^{\text {Tum-1 }}$ ), act5c-GAL4/CyO (BL\#4414), UAS-myr-RFP/CyO (BL\#7118) and hml-GAL4 (BL\#30139) were obtained from Bloomington Drosophila Stock Center, Bloomington, IN. UAS-hipk ${ }^{R N A i}$ (VDRC\#108254) was obtained from Vienna Drosophila Resource Center, Vienna, Austria. Also used were dpp-GAL4/TM6B (StaehlingHampton et al., 1994), os,y (a gift from Norbert Perrimon), w;UAS-Stat92EMyc/Cyo,wg-lacZ (a gift from Sol Sotillos) (Sotillos et al., 2013), PD-lacZ (a gift from Henry Sun; referred to as upd-lacZ hereon after) (Tsai and Sun, 2004), ywhsflp,tubGAL4,UAS-GFP,6X MYC-NLS; UAS-y+;tub-GAL80,FRT2A/TM6B (a gift from Gary Struhl), ywhsflp ${ }^{122}$;sp/Cyo;TM2/TM6B, UAS-HA-hipk ${ }^{1 M}$, UAS-HA-hipk ${ }^{3 M}$, hipk $^{4}$,FRT79/TM6B (Lee et al., 2009a), UAS-HA-hipk ${ }^{K 221 R}$-attP40 (kinase dead Hipk; hereafter referred to as UAS-HA-hipk ${ }^{K D}$-attP40) (Chen and Verheyen, 2012), and UASHA-hipk ${ }^{W T}$-attP40 (made in this study). act5c-GAL4/Cyo and UAS-myr-RFP/CyO were recombined to generate act5c-GAL4,UAS-myr-RFP/CyO. hipk ${ }^{4}$, FRT79/TM6B and 10xstat92E-GFP/TM6B were recombined to generate hipk ${ }^{4}$, FRT79, 10xstat92EGFP/TM6B.

Generation of transgenic fly stocks: DNA cloning was performed by Ziwei Ding of the SFU Molecular Biology Service Centre. pCMV-HA-Hipk (Lee et al., 2009a) was used as the source of HA-Hipk. The EcoRI site of pUASt-attB was mutated to a Smal site, and HA-hipk ${ }^{W T}$ was inserted into this site. HA-hipk ${ }^{W T}$-attB was inserted into the attP40 locus generating the fly strain UAS-HA-hipk ${ }^{W T}$-attP40 (Best Gene, Chino Hills, $\mathrm{CA})$.

Clonal analysis: Somatic clones were generated by crossing $h$ sflp $^{122, ; U b i-R F P, F R T 79}$ to either 10XStat92E-GFP; hipk ${ }^{4}$,FRT79/TM6B, or upd-lacZ;; hipk ${ }^{4}$, FRT79/TM6B.

Progeny were heat shocked at $38^{\circ} \mathrm{C}, 48$ hours after egg laying for 90 minutes. MARCM clones were generated by crossing ywhsflp ${ }^{122}$;act5c-GAL4,UAS-myr- 
RFP/CyO;tub-GAL80,FRT2A/TM6B (RFP MARCM79) to either hipk $^{4}$,FRT79, 10xstat92E-GFP/TM6B, UAS-HA-hipk ${ }^{W T}$-attP40; hipk $^{4}$,FRT79, 10xstat92EGFP/SM6a TM6B, or UAS-HA-hipk ${ }^{K D}$-attP40; hipk $^{4}$,FRT79, 10xstat92E-

GFP/SM6a TM6B. Progeny were heat shocked at $38^{\circ} \mathrm{C}, 48$ hours after egg laying for 90 minutes and were subsequently raised at $29^{\circ} \mathrm{C}$.

Immunocytochemistry and microscopy: L3 imaginal discs were dissected and stained using standard protocols, and where possible we analyzed equal to or greater than 20 discs per genotype; the exception to this was the MARCM experiments in Fig. 3 and S3 which were particularly sickly and thus had lower n-values. Detailed information regarding antibodies used and methods of microscopy can be found in the Supplementary Materials.

Eye size comparison for os assay: 10 images were acquired for TM3/TM6B, hipk ${ }^{4} / T M 6 B$, os;;MKRS/TM6B, and os;;hipk ${ }^{4} / T M 6 B$ adult eyes. The area of each eye was measured in pixels using Photoshop, and the values were subjected to a student's t-test.

hop $^{\text {Tum-l }}$ lethality and tumor frequency assays: The lethality assay in Fig. 2 was performed by crossing 50 females and 15 males from each stock (hop ${ }^{\text {Tum- }}$

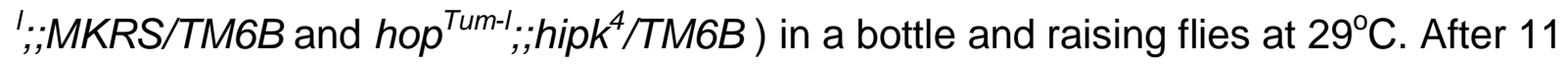
days, all pupae were removed from the walls of the bottles and were ranked as either 'early pupal lethal' (had no recognizable adult structures), 'late pupal lethal' (pharate adults), or 'eclosed adult' (see examples of each rank in Fig. 2F-H). The lethality assay in Fig. S2A-D was performed by crossing 8 females (hop ${ }^{\text {Tum-l }} /($ FM7);hml-GAL4) to 6 males (either $w^{1118} / Y$ or UAS-hipk ${ }^{R N A j}$ ) in a vial and raising flies at $29^{\circ} \mathrm{C}$. Progeny were scored using the same methods as the previous lethality assay (for scoring examples see Fig. S2A-C). The tumor frequency assay in Fig. S2E-H was performed by crossing 8 females (hop ${ }^{\text {Tum-l }} /\left(\right.$ FM7); $h m$ l-GAL4) to 6 males (either $w^{1118} / Y$ or $X / Y ; U A S-h i p k^{R N A i}$ ) in 
a vial and raising flies at $25^{\circ} \mathrm{C}$. After 13 days, male progeny were scored into the following classifications: 'class 1' (flies had greater than 5 tumors ranging in size from small to large), 'class 2' (more than 5 small to medium tumors were present), and 'class 3' (less than 5 small tumors were present) (see examples of each class in Fig. S2E-G).

Hemocyte counts: Prior to hemolymph collection, L3 larvae were washed thoroughly with $1 \mathrm{X}$ PBS, dried, and placed in a glass dissection well containing $5 \mu \mathrm{L}$ of $1 \mathrm{X}$ PBS. The larval cuticle was carefully punctured with forceps and hemolymph was allowed to drain into the well. Each sample contains the hemolymph from two larvae in $5 \mathrm{uL}$ of $1 \mathrm{X}$ PBS. Hemolymph was then smeared onto a poly-D-lysine coated slide and air-dried. Cell smears were washed with 3.7\% formaldehyde for 5 minutes, washed with PBS, and stained with DAPI. For each sample $(n=10), 5$ cell counts were performed and means of the 5 cell counts were plotted; values were subjected to a student's t-test.

Proximity Ligation Assay (PLA): PLA was performed on L3 wing discs according to standard protocols (Wang et al., 2014) with the following exceptions: discs were fixed with $4 \%$ formaldehyde for 15 minutes and discs were blocked with $1 \%$ normal donkey serum. A PLA against HA and GFP was used as a negative control. A subset of the discs were stained for HA to ensure that Hipk was expressed.

\section{Acknowledgements:}

We are grateful to N. Perrimon, H. Sun, S. Sotillos, Bloomington Drosophila Stock Center (NIH P40OD018537), and Developmental Studies Hybridoma Bank for providing fly strains and antibodies. Also, we thank Z. Ding for help in creating the HAhipkWT-attP40 construct, and A. Kadhim for help with crosses. This work was funded by an operating grant from the Canadian Institutes of Health Research. NBW was supported by a PGS-M fellowship award from N.S.E.R.C.

Competing Interests: The authors declare no competing of financial interests. 
Author contributions: Conception and design JB, NW, EV. Acquisition of data JB, NW. Analysis and interpretation of data JB, NW, EV. Drafting or revising the article JB, NW, EV.

Funding: This research was funded by a Canadian Institutes of Health Research Grant [MOP 97835].

\section{Figure Legends}

Figure 1. Hipk induces hemocyte derived melanotic tumors. (A) A control $d p p>$ GFP L3 larva. (B) Stationary melanized masses are observed in $65 \%$ of $d p p>H A$ hipk $^{3 M}+$ GFP larvae (blue arrowheads; $\mathrm{n}=40$ ) and (C) persist when apoptotic cell death is inhibited in $d p p>H A-h i p k^{3 M}+P 35+G F P$ larvae. (D) The abdomen of a control $h m l>G F P$ fly. (E) Melanized tumors are present in $h m l>H A-h i p k^{3 M}$ flies (blue arrowheads). Spermathecae were not counted (magenta arrowhead). (F) Quantification of the number of tumors scored in from the dissected abdomens of flies shown in (D) and (E), $n=36$ for both groups. Smears of total hemolymph collected from (G) $h m l>2 X G F P$ and (H) $h m l>H A-h i p k^{3 M}+G F P$ L3 larvae. (I) Quantification of mean number of hemocytes counted from genotypes in $(\mathbf{G})$ and $(\mathbf{H})$. Each data point represents the mean of 5 cell counts from one sample, $h m l>2 x G F P$ ( $\mathrm{n}=10$ samples, $\mathrm{n}=50$ cell counts) and $h m />H A-h i p k^{3 M}+G F P(\mathrm{n}=10$ samples, $\mathrm{n}=50$ cell counts), $\mathrm{P}<0.0001$. Scale bars equal $10 \mu \mathrm{m}$.

Figure 2: Heterozygous loss of hipk suppresses $h o p^{\text {Tum-l }}$ induced lethality. (A,B) At $29^{\circ} \mathrm{C}$ hop ${ }^{\text {Tum-l }}$ causes the formation of melanized tumors (A; arrowheads) and (B) results in larval and/or pupal lethality. (C,D) Heterozygous loss of hipk suppresses the tumor frequency (C; arrowheads) and (D) though some hop ${ }^{\text {Tum-l } ; ; \text {; } h i p k^{4} / T M 6 B}$ flies die in the early pupal stage, many reach the late pupal stage. (E) Quantification of the hop ${ }^{\text {Tum- }}$ ' lethality test in (A-D); hop ${ }^{\text {Tum-l } ; ; M K R S / T M 6 B ~(n=148) ~ a n d ~ h o p ~}{ }^{\text {Tum-l } ; ; \text {;ipk }}{ }^{4} / T M 6 B$ $(n=193)$. The hop ${ }^{\text {Tum-l }}$ lethality assay was phenotypically ranked into three categories: (F) represents category 'early pupal lethal', where no adult structures are detectable, (G) represents the 'late pupal lethal' class, where adult structures are visible but the fly does not eclose, and $(\mathbf{H})$ represents the class 'eclosed adult'. 


\section{Figure 3: hipk promotes and is required for JAK/STAT signaling, downstream of} upd. (A) A control L3 wing disc showing the expression domain of the reporter 10xStat92E-GFP. (B) Expression of 10xStat92E-GFP is perturbed in hipk ${ }^{4}$ mutant clones marked by the absence of RFP (arrowheads) $(n=20)$. (C) Expressing UAS-HAhipk $^{W T}$ within hipk ${ }^{4}$ MARCM clones (act>>HA-hipk ${ }^{W T}$; hipk $^{4}$ ) restores and can elevate 10xStat92E-GFP levels ( $n=10)$. (D) 10xStat92E-GFP levels are not restored within act>>HA-hipk ${ }^{K D}$; hipk $^{4}$ clones ( $\mathrm{n}=5$ ). (E) Increases in 10xStat92E-GFP expression are observed in dpp>HA-hipk ${ }^{3 M}$ wing discs (arrowheads) raised at $25^{\circ} \mathrm{C}(\mathrm{n}=20)$. (F) A control wing disc showing the expression domain of upd-lacZ. (G) At $25^{\circ} \mathrm{C}$, upd-lacZ appears unchanged in $d p p>H A$-hipk ${ }^{3 M}$ wing discs $(\mathrm{n}=20)$. (H) upd-lac $Z$ is expressed at the posterior center of the L3 eye-antennal control disc. (I) Loss of hipk, in negatively marked RFP clones, does not affect upd-lac $Z$ (arrowhead) $(n=20)$. Scale bars equal $10 \mu \mathrm{m}$.

Figure 4: HA-Hipk and MYC-Stat92E physically interact in the wing imaginal disc. PLAs were performed on L3 wing imaginal discs. (A) A negative control disc probed with anti-HA and anti-GFP antibodies did not yield a positive signal $(n=17)$. (B) $d p p>H A-h_{i p k}{ }^{1 M}+M Y C$-stat92E discs stained for HA show that Hipk is present in the same discs used for the PLA experiments. (C,D) A positive PLA signal is present along the dpp domain in dpp>HA-hipk ${ }^{1 M}+M Y C$-stat92E discs that were probed with HA and MYC ( $n=54)$. The boxed region in (C) represents the zoomed-in region in (D). Scale bars equal $10 \mu \mathrm{m}$. 


\section{References:}

Amoyel, M., Anderson, A. M. and Bach, E. A. (2014). JAK/STAT pathway dysregulation in tumors: a Drosophila perspective. Semin. Cell Dev. Biol. 28, 96103.

Bach, E. A., Ekas, L. A., Ayala-Camargo, A., Flaherty, M. S., Lee, H., Perrimon, N. and Baeg, G.-H. (2007). GFP reporters detect the activation of the Drosophila JAK/STAT pathway in vivo. Gene Expr. Patterns 7, 323-31.

Chen, J. and Verheyen, E. M. (2012). Homeodomain-interacting protein kinase regulates yorkie activity to promote tissue growth. Curr. Biol. 22, 1582-1586.

Chen, Q., Giedt, M., Tang, L. and Harrison, D. A. (2014). Tools and methods for studying the Drosophila JAK/STAT pathway. Methods 68, 160-72.

Dearolf, C. R. (1998). Fruit fly "leukemia”. Biochim. Biophys. Acta 1377, M13-23.

Fleischmann, K. K., Pagel, P., Schmid, I. and Roscher, A. a (2014). RNAi-mediated silencing of MLL-AF9 reveals leukemia-associated downstream targets and processes. Mol. Cancer 13, 27.

Hanratty, W. P. and Dearolf, C. R. (1993). The Drosophila Tumorous-lethal hematopoietic oncogene is a dominant mutation in the hopscotch locus. Mol Gen Genet 238, 33-37.

Hanratty, W. P. and Ryerse, J. S. (1981). A genetic melanotic neoplasm of Drosophila melanogaster. Dev. Biol. 83, 238-249.

Harrison, D., Binari, R. and Nahreini, T. (1995). Activation of a Drosophila Janus kinase (JAK) causes hematopoietic neoplasia and developmental defects. EMBO J. 14, 2857-2865.

Hay, B., Wolff, T. and Rubin, G. (1994). Expression of baculovirus P35 prevents cell death in Drosophila. Development 2129, 2121-2129.

Jones, A., Kreil, S., Zoi, K. and Waghorn, K. (2005). Widespread occurrence of the JAK2 V617F mutation in chronic myeloproliferative disorders. Blood 106, 21622169. 
Kim, Y. H., Choi, C. Y., Lee, S. J., Conti, M. A. and Kim, Y. (1998). Homeodomaininteracting protein kinases, a novel family of co-repressors for homeodomain transcription factors. J. Biol. Chem. 273, 25875-25879.

Kralovics, R., Passamonti, F., Buser, A. S., Teo, S., Tiedt, R., Passweg, J. R., Tichelli, A., Cazzola, M. and Skoda, R. C. (2005). A gain-of-function mutation of JAK2 in myeloproliferative disorders. N. Engl. J. Med. 352, 1779-1790.

Lacronique, V. (1997). A TEL-JAK2 Fusion Protein with Constitutive Kinase Activity in Human Leukemia. Science (80-. ). 278, 1309-1312.

Lee, W., Andrews, B. C., Faust, M., Walldorf, U. and Verheyen, E. M. (2009a). Hipk is an essential protein that promotes Notch signal transduction in the Drosophila eye by inhibition of the global co-repressor Groucho. Dev. Biol. 325, 263-272.

Lee, W., Swarup, S., Chen, J., Ishitani, T. and Verheyen, E. M. (2009b). Homeodomain-interacting protein kinases (Hipks) promote WntWg signaling through stabilization of beta-catenin/Arm and stimulation of target gene expression. Development 136, 241-251.

Levine, R. L., Wadleigh, M., Cools, J., Ebert, B. L., Wernig, G., Huntly, B. J. P., Boggon, T. J., Wlodarska, I., Clark, J. J., Moore, S., et al. (2005). Activating mutation in the tyrosine kinase JAK2 in polycythemia vera, essential thrombocythemia, and myeloid metaplasia with myelofibrosis. Cancer Cell 7, 38797.

Luo, H., Hanratty, W. and Dearolf, C. (1995). An amino acid substitution in the Drosophila hopTum-I Jak kinase causes leukemia-like hematopoietic defects. EMBO J. 14, 1412-1420.

Luo, H., Rose, P., Barber, D., Hanratty, W. P., Lee, S., Roberts, T. M., D'Andrea, a D. and Dearolf, C. R. (1997). Mutation in the Jak kinase JH2 domain hyperactivates Drosophila and mammalian Jak-Stat pathways. Mol. Cell. Biol. 17, 1562-1571.

Matsuo, R., Ochiai, W., Nakashima, K. and Taga, T. (2001). A new expression cloning strategy for isolation of substrate-specific kinases by using phosphorylation site-specific antibody. J. Immunol. Methods 247, 141-151.

Ohtsu, N., Nobuhisa, I., Mochita, M. and Taga, T. (2007). Inhibitory effects of homeodomain-interacting protein kinase 2 on the aorta-gonad-mesonephros hematopoiesis. Exp. Cell Res. 313, 88-97. 
Poon, C. L. C., Zhang, X., Lin, J. I., Manning, S. A. and Harvey, K. F. (2012). Homeodomain-interacting protein kinase regulates Hippo pathway-dependent tissue growth. Curr. Biol. 22, 1587-1594.

Rawlings, J. S., Rennebeck, G., Harrison, S. M. W., Xi, R. and Harrison, D. A. (2004). Two Drosophila suppressors of cytokine signaling (SOCS) differentially regulate JAK and EGFR pathway activities. BMC Cell Biol. 5, 38.

Rinaldo, C., Siepi, F., Prodosmo, A. and Soddu, S. (2008). HIPKs: Jack of all trades in basic nuclear activities. Biochim. Biophys. Acta - Mol. Cell Res. 1783, 21242129.

Sinenko, S. a and Mathey-Prevot, B. (2004). Increased expression of Drosophila tetraspanin, Tsp68C, suppresses the abnormal proliferation of ytr-deficient and Ras/Raf-activated hemocytes. Oncogene 23, 9120-9128.

Söderberg, O., Gullberg, M., Jarvius, M., Ridderstråle, K., Leuchowius, K.-J., Jarvius, J., Wester, K., Hydbring, P., Bahram, F., Larsson, L.-G., et al. (2006). Direct observation of individual endogenous protein complexes in situ by proximity ligation. Nat. Methods 3, 995-1000.

Sotillos, S., Krahn, M., Espinosa-Vazquez, J. M. and Hombria, J. C.-G. (2013). Src kinases mediate the interaction of the apical determinant Bazooka/PAR3 with STAT92E and increase signalling efficiency in Drosophila ectodermal cells. Development 140, 1507-1516.

Staehling-Hampton, K., Jackson, P. D., Clark, M. J., Brand, A. H. and M, H. F. (1994). Specificity of bone morphogenetic protein-related factors: cell fate and gene expression changes in Drosophila embryos induced by decapentaplegic but not 60A. Cell growth Differ. 5, 585-593.

Swarup, S. and Verheyen, E. M. (2011). Drosophila homeodomain-interacting protein kinase inhibits the Skp1-Cul1-F-box E3 ligase complex to dually promote Wingless and Hedgehog signaling. Proc. Natl. Acad. Sci. U. S. A. 108, 9887-9892.

Tsai, Y. and Sun, Y. H. (2004). Long-range effect of upd, a ligand for Jak/STAT pathway, on cell cycle in Drosophila eye development. Genesis 39, 141-53.

Wang, S. J. H., Tsai, A., Wang, M., Yoo, S., Kim, H., Yoo, B., Chui, V., Kisiel, M., Stewart, B., Parkhouse, W., et al. (2014). Phospho-regulated Drosophila adducin is a determinant of synaptic plasticity in a complex with Dlg and PIP2 at the larval neuromuscular junction. Biol. Open 3, 1196-1206. 
bioRxiv preprint doi: https://doi.org/10.1101/058156; this version posted June 10, 2016. The copyright holder for this preprint (which was not certified by peer review) is the author/funder. All rights reserved. No reuse allowed without permission.

Yan, R. and Luo, H. (1996). A JAK-STAT pathway regulates wing vein formation in Drosophila. Proc. Natl. Acad. Sci. U. S. A. 93, 5842-5847. 
。
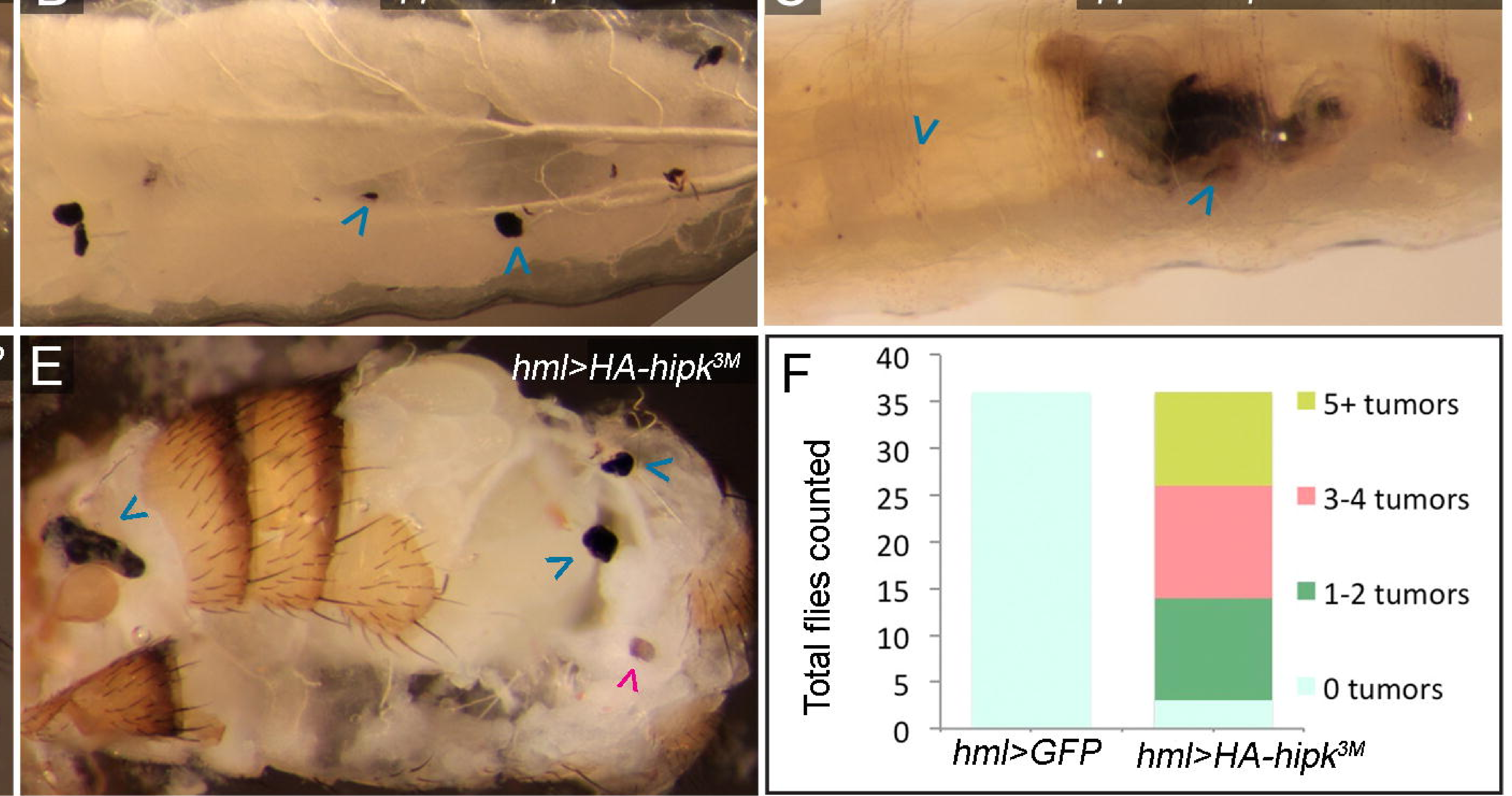

\section{G} $h m>$ GFP E
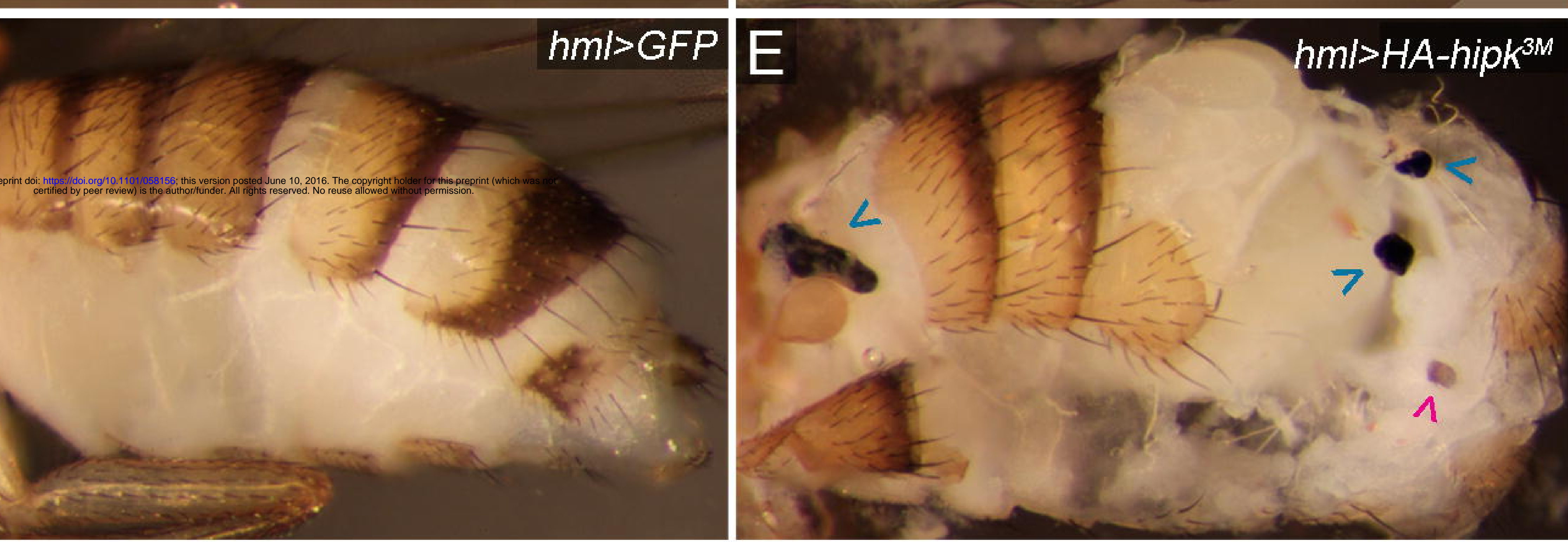

GFP $h m l>2 x G F P$
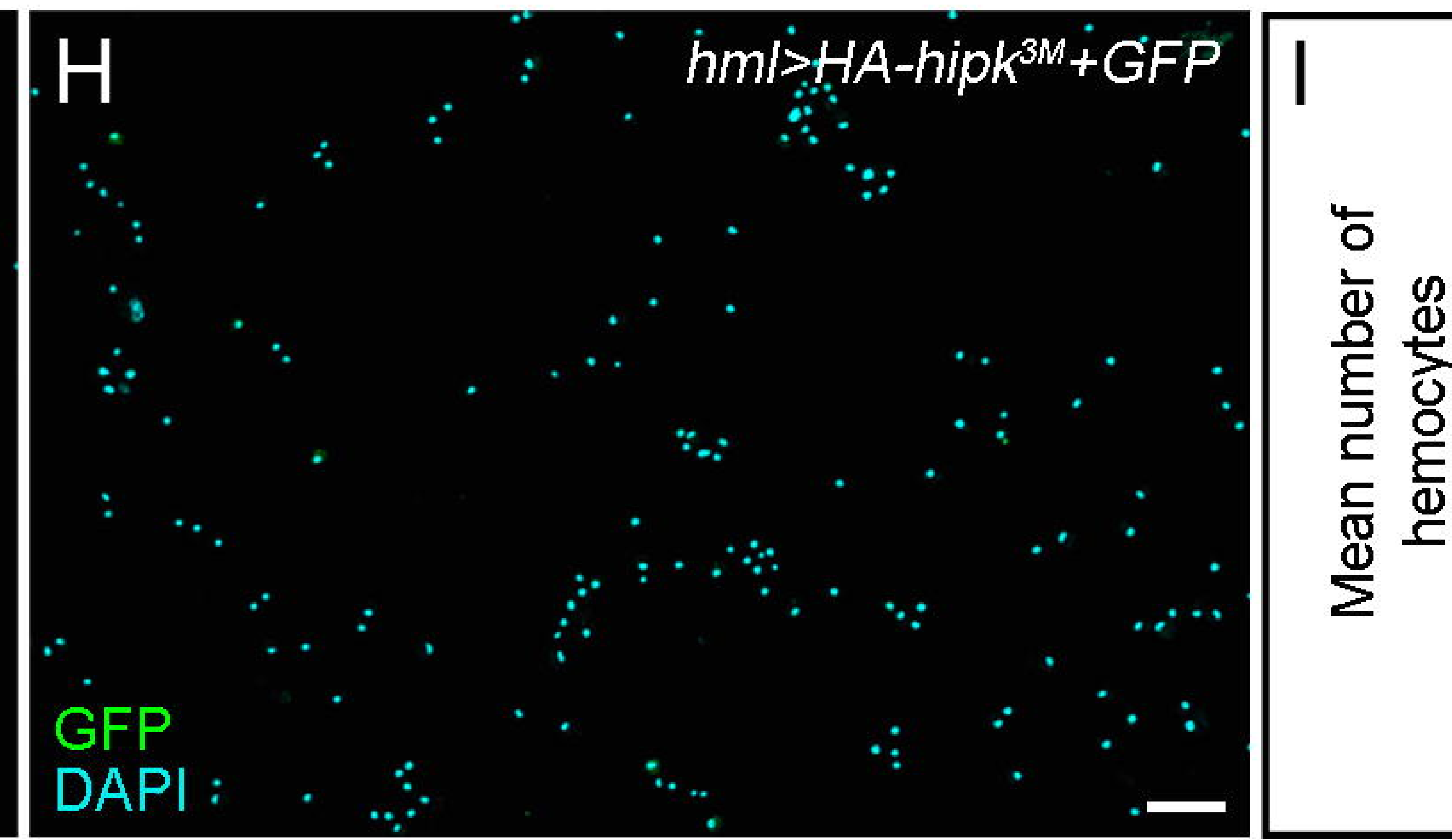

300
250
200
150
100
50

$300-$
$50-$
$50-$
$00-$
0
0

$\infty$

O $\triangle$

$h \mathrm{ml}$ $h m l>H A-h^{2} k^{3 m}$ $2 x G F P$ $+G F P$ 

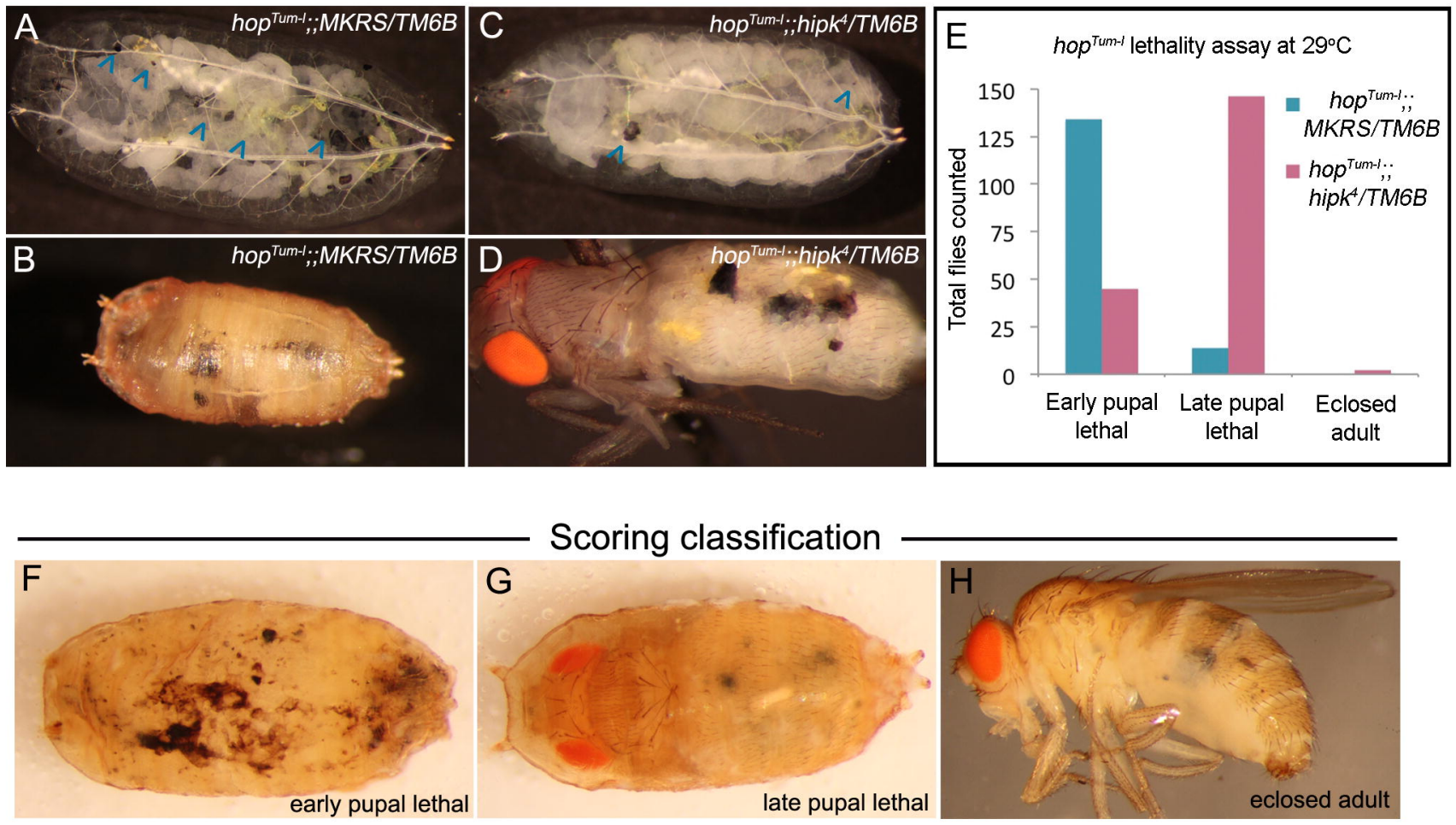

\section{Scoring classification}
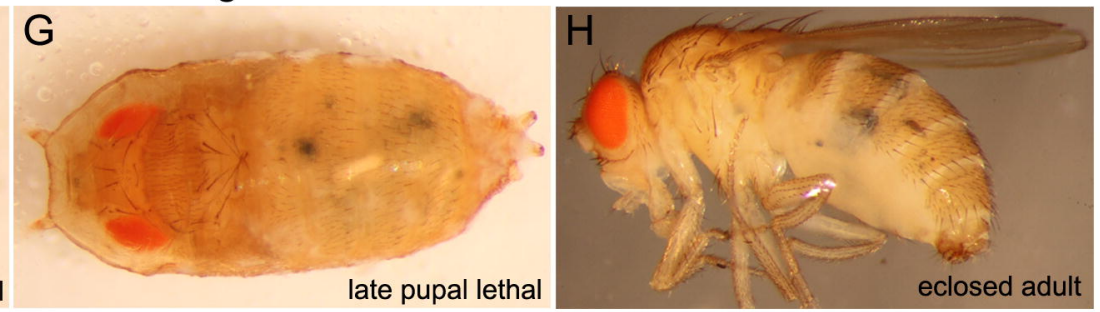

Figure 2 


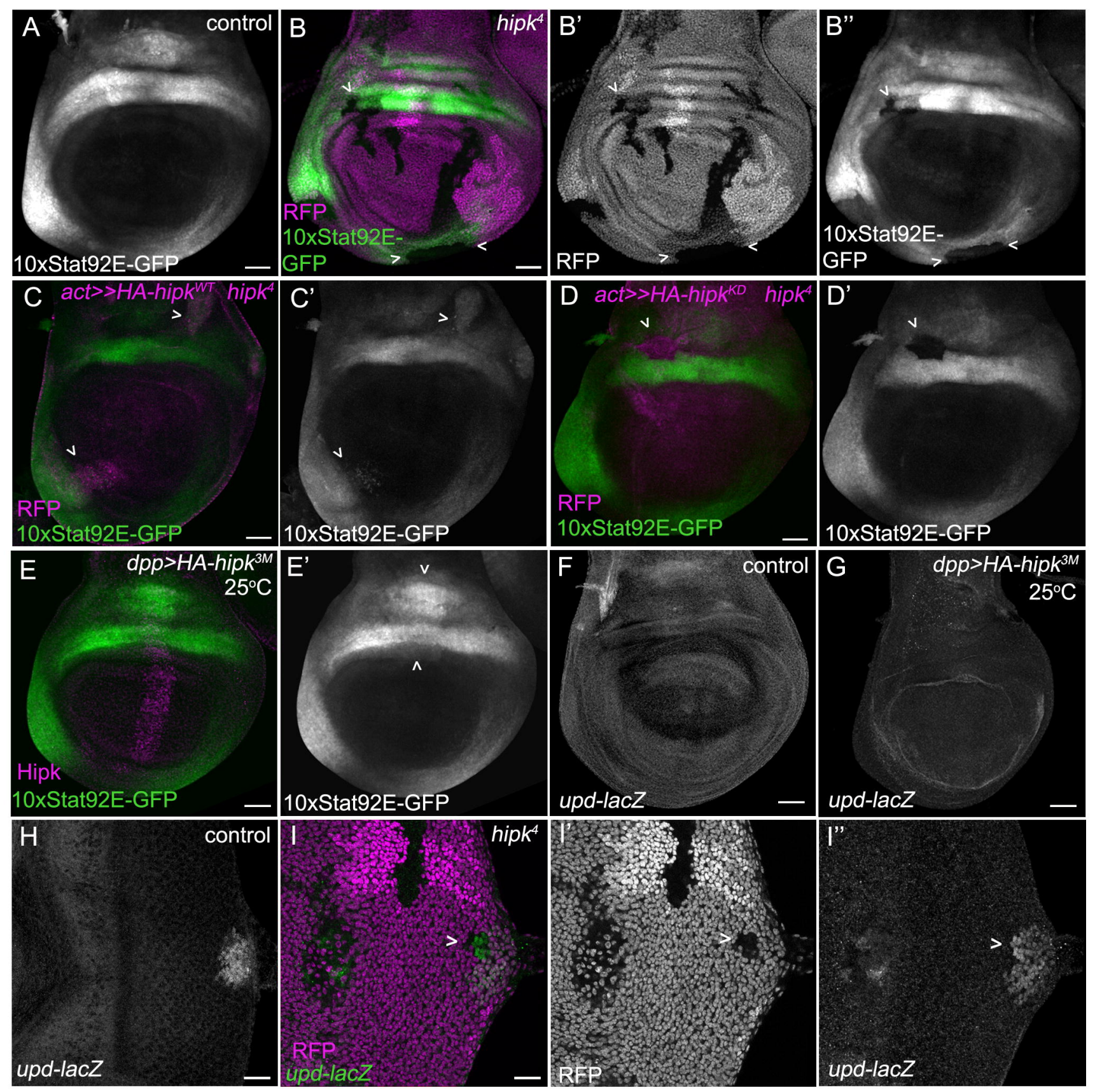

Figure 3 


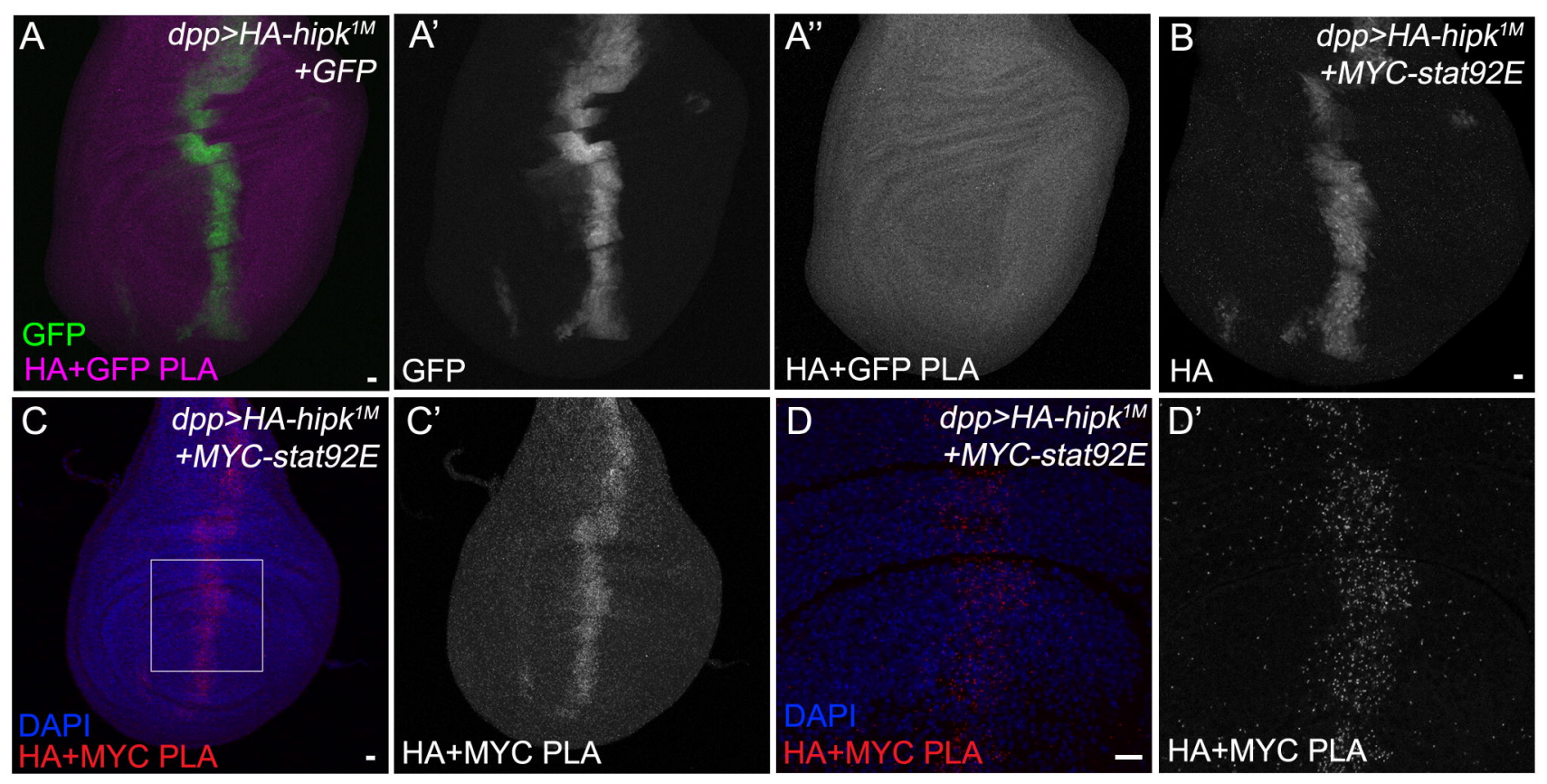

Figure 4 